\title{
MIAM: A ROBOT ORIENTED MOBILE INTELLIGENT AGENT MODEL
}

\author{
Wu Shandong and Chen Yimin \\ School of Computer Engineering and Science, Shanghai University
}

\begin{abstract}
This paper proposes a robot oriented Mobile Intelligent Agent Model-MIAM, composed of core function, mobile ability, intelligent engine and communication interfaces, which provide flexible multi-mode robot control solutions for intelligent control and remote control.
\end{abstract}

Key words: robot, agent, MIAM, intelligent control, remote control

\section{INTRODUCTION}

Intelligent control and remote control are two important control modes for robot. The software agent technique provides an effective way for constructing multi-modes robot control system [1]. Focusing on providing high adaptability, this paper proposed a robot oriented Mobile intelligent Agent Model-MIAM and, the architecture and an applications example are introduced in detail.

\section{MIAM-MOBILE INTELLIGENT AGENT MODEL}

MIAM model is illustrated in Fig. 1. MIAM is a compound architecture, with no particular emphasis on intelligent agent or mobile agent. MIAM is designed for robot intelligent control and remote control and it is composed of four parts namely, core function, mobile ability, intelligent engine and communication interface. Below is a detailed introduction of the architecture of MIAM. 


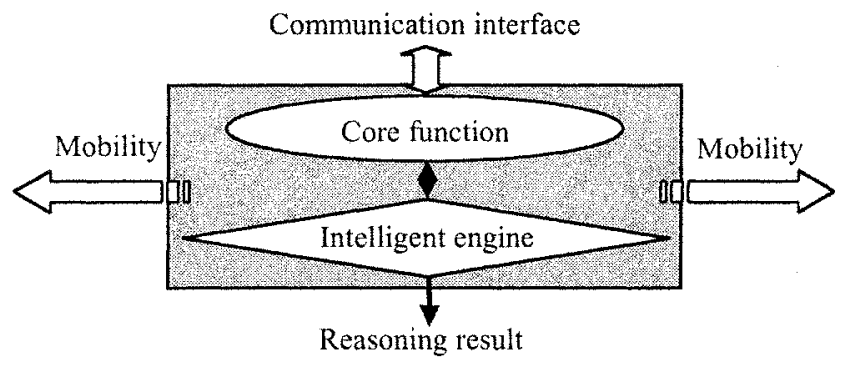

Figure I. MIAM model

\subsection{Core function}

The core function is specified function of the agent itself, that is to say, what it could do naturally. In a robot control system there are several kinds of agent as follows with different core functions.

- Target agent. To perform target tasks directly such as conducting welding and conveying.

- Simulate agent. To trace and simulate the robot's motion process.

- Status agent. To monitor the motion and provide real time status message.

- Message agent. To send or receive none-real time messages.

- Collaboration agent. To manage working agents in a group as a chief.

\subsection{Mobile ability}

The free mobility of agent on network leads to more flexibility of the robot control system. For a remote robot object, mobile agent is able to move freely to the desired computer node to execute robot control task. User and robot are located on a fixed position. However, the mobile ability of agent provides a strong base for remote robot control mode.

The MIAM based remote robot control acts as following: the user sets up a task model, organizes various agents to form an appropriate agent team, and assigns subtasks to agents and configures the collaboration rules and relations, and then dispatches agent team to conduct robot control. Agents move automatically according to the itinerary initialized to the destination node. Agent could clone itself as need arises. During work time, the collaboration relations among agents may be changed or reorganized dynamically, usually under coordination of the collaboration agent. After finishing the task, agent may either return to the user, dispose of itself, or set stored in a new medium waiting to be re-actived. However, user could retract any dispatched agent home at any time. 


\subsection{Intelligent engine}

The intelligent engine provides agent with intelligence. The advanced characteristics of agent depend on the intelligence in a higher degree. Our desire is that agent could as highly be intelligent as a person and be autonomous in solving the problems if faces, while asking little help from people. For MIMA model, we made agent possess both forward and backward directions intelligent reasoning abilities. The forward reasoning would make robot fully self-adaptability, that is to say, at any time and situation agent could reason out the next step's action. On the other hand, the backward reasoning is suitable for resolving questions inversely.

There exist multiple agents in the whole robot control system. Each single agent has its own local intelligent reasoning engine, which provides intelligence support for its own functions. For the agent collaboration, a global reasoning engine is required, which provides information sharing and fusing to support group intelligence. The global agent collaboration and reasoning engine is lightweight but with higher intelligence, which aims at resolving and processing the vital global questions, correspondingly, more partial and detailed questions are throw to agents for further processing.

\subsection{Communication interface}

In multi-agent system, communication between agents is a natural requirement and the basis of agent collaboration. MIAM agent is equipped with two kinds of communication interface. One is the Point to Point (P2P) interface, which enables agent to communicate directly and quickly with another desired agent. The other is a public interface for information sharing among multiple agents. Because each agent has its own knowledge, ability and apperception from environment, an ISC (Information Share Centre) is required in the system to make agents share or interchange information. Information sharing and fusing are the key basics for the agent collaboration, accordingly, besides providing and broadcasting static message, the ISC should be combined with agent collaboration rules.

Besides interface, another key issue remaining is agent communication language. A perfect protocol can provide standardized agent interface. Some famous protocols such as KQML, KIF and ACL are available.

\section{MIAM BASED APPLICATION EXAMPLE}

According to the MIAM model introduced above, taking PT500 robot as the control objects, we built a multiple modes robot control system. A user 
can organize different kinds of agent dynamically and purposefully to form a work team to perform robot intelligent control or remote control. Below is a brief introduction of MIAM implementation in this system.

The kernel function of MIAM is aimed at controlling PT500 robot to motion and assemble. The control functions mainly include robot program interpreting, instruction library, control card driver, etc. The detailed robot functions are introduced in the related reference [2].

The MIAM communication language is KQML. The communication contents include control command and robot joints position data. The later is used to simulate robot motion, or may be transferred to the global reasoning engine to analyze and predict whether collision would happen between two robots. The former include the commands such as STOP, PAUSE robot, which come from the user or the reasoning engine.

The reasoning engine of MIAM is implemented by Jess [3]. Because the robot instructions of PT500i s obtained through teaching, the main intelligent engine in MIAM is forward reasoning. Two reasoning scripts are designed for single robot motion and the collaborative control of two robots.

The mobility of MIAM is implemented by Aglet [4]. Each aglet is equipped with a task interface which is used to assign robot program, viz. a set of robot instructions, in the remote robot control model. Aglet will move to desired remote robot nodes to interpret and execute the robot program to drive the robot.

\section{CONCLUSION}

This paper proposes a Mobile Intelligent Agent Model and constructed a multi-mode robot control system for PT500 robot applying MIAM. MIAM is suitable for robot intelligent control and remote control, and the experience shows that the control system has a favorable control performance.

\section{REFERENCES}

1. P. Bellavista, A. Corradi, C. Stefanelli, Mobile Agent Middleware for Mobile Computing, IEEE Computer, Vol. 34, No. 3, pages 73-81, March 2001

2. WU Shan-dong, CHEN Yi-min, HE Yong-yi, Development Technique of High Level Software System for Industrial Robot Controller Base on WIN 9X/NT, Journal of Shanghai University, Dec. 2001, Vol.7, No.6, pp. 532-535

3. Ernest Friedman-Hill, Jess, http://herzberg.ca.sandia.gov/jess/main.html, Sandia National Laboratories, Livermore, CA, 1995

4. IBM Aglets, http://www.trl.ibm.co.jp/aglets/ 